\title{
A Plant Ancestral Polo-Like Kinase Sheds Light on the Mystery of the Evolutionary Disappearance of Polo-Like Kinases in the Plant Kingdom
}

\author{
Erika Okamura, Takuya Sakamoto, Tatsuki Sasaki and Sachihiro Matsunaga* \\ Department of Applied Biological Science, Faculty of Science and Technology, Tokyo University of Science, \\ 2641 Yamazaki, Noda, Chiba 278-8510, Japan
}

Received January 24, 2017; accepted February 6, 2017

\begin{abstract}
Summary Polo-like kinase (PLK) is a serine/threonine kinase involved in the formation of the mitotic spindle and the maturation of centrosomes. PLKs are highly conserved in the animal and fungi kingdoms but have disappeared in land plants. Plant PLK orthologs have been found in green algae, red algae and a filamentous terrestrial alga, Klebsormidium flaccidum, which can adapt to both fresh water and land environments. Our evolutionary analyses suggest that plants evolutionally lost the PLK ortholog before the divergence of land plants. Our localization analyses of the PLK ortholog in Cyanidioschyzon merolae demonstrated that it is localized in spindle poles and on mitochondrial polar microtubules, suggesting that plant ancestral PLK functions in the formation of mitotic spindles like animal and fungus PLK orthologs.
\end{abstract}

Key words Polo-like kinase, Plant evolution, Mitotic spindle, Algae, Cyanidioschyzon merolae, Klebsormidium flaccidum.

Various protein kinases play important roles in the regulation of cell division in eukaryotes (Nurse 1990, Dewitte and Murray 2003). Polo-like kinase (PLK) functions as a key regulator of the cell cycle (Zitouni et al. 2014) and the loss of PLK induces tumorigenesis (de Cárcer et al. 2011b). PLK is classified into three subfamilies; the PLK1, PLK2 and PLK4/SAK subfamilies. All PLKs have a catalytic serine/threonine kinase domain in the N-terminal region and one (for the PLK4/SAK subfamily) or two (for the PLK1 and PLK2 subfamilies) polo-box domains (PBDs) (de Cárcer et al. 2011b, Zitouni et al. 2014). The number of PLKs and PLK subfamilies in the genome differ among eukaryotic species. Yeasts have only one PLK, such as Cdc5 in Saccharomyces cerevisiae and plo1 in Schizosaccharomyces pombe, whereas most metazoans have several PLKs (Barr et al. 2004, de Cárcer et al. 2011b, Zitouni et al. 2014). Drosophila melanogaster has polo in the PLK1 subfamily (Sunkel and Glover 1988) and SAK in the PLK4/SAK subfamily (Fode et al. 1994). Humans have five PLK paralogs including PLK1, PLK4, and PLK2, 3 and 5 in the PLK2 subfamily (de Cárcer et al. 2011b).

PLK1 is the ancestral PLK and the PLK2 and PLK4/SAK subfamilies originated after gene duplication (de Cárcer et al. 2011b). In animal cells, PLK1 is localized in centrosomes during G2 phase, at kinetochores and spindle poles from prometaphase to metaphase, and

\footnotetext{
*Corresponding author, e-mail: sachi@rs.tus.ac.jp

DOI: $10.1508 /$ cytologia.82.261
}

at the central spindle from anaphase to telophase (Barr et al. 2004). PLK1 has multiple functions throughout the cell cycle, including centrosome maturation, mitotic entry, spindle formation and cytokinesis (Carvalho-Santos et al. 2010, Zitouni et al. 2014). PLK2 is localized in centrioles and plays roles in centriole replication and the G1/S transition (Ma et al. 2003, Cizmecioglu et al. 2008, Zitouni et al. 2014). PLK3 is located in nuclei, centrosomes and mitotic spindles, and is involved in DNA replication and the G1/S and G2/M transitions (Zimmerman and Erikson 2007, Wang et al. 2002, Zitouni et al. 2014). Some vertebrate species, including humans and mice, have PLK5 (de Cárcer et al. 2011b, Zitouni et al. 2014). Human PLK5, which lacks part of the kinase domain, is involved in neuron differentiation (Wang et al. 2002, de Cárcer et al. 2011a). Mouse subnuclear PLK5, which has a full kinase domain, contributes to DNA replication and the G1/S transition (Andrysik et al. 2010). The divergence event between the PLK4/SAK and PLK1 subfamilies occurred before the divergence between metazoans and fungi (Zitouni et al. 2014). The PLK4/SAK subfamily is conserved in metazoans and some fungi (Carvalho-Santos et al. 2010). PLK4 is localized in centrosomes and plays a crucial role in centriole replication and flagellar formation (Kleylein-Sohn et al. 2007, Bettencourt-Dias et al. 2005, Zitouni et al. 2014).

Although PLKs are conserved among most eukaryotic species, no PLK homolog is found in land plants (Lowery et al. 2005, Carvalho-Santos et al. 2010, de Cárcer et al. 2011b, Zitouni et al. 2014). In contrast, some algae 
including Ostreococcus tauri, Chlamydomonas reinhardtii and Cyanidioschyzon merolae have a PLK ortholog (Hodges et al. 2010, Carvalho-Santos et al. 2010, Zitouni et al. 2014) but their functions remain unknown.

C. merolae is a unicellular alga found in strongly acidic hot springs (Matsuzaki et al. 2004) for which all DNA sequences in the nucleus (16.5 Mbp), mitochondrion and chloroplast are available (Ohta et al. 1998, 2003, Matsuzaki et al. 2004, Nozaki et al. 2007). C. merolae has only 4775 genes (Nozaki et al. 2007) and a single mitochondrion, chloroplast and peroxisome, indicating that the subcellular structure is simple (Imoto et al. 2013). Its organelle division can be synchronized by light and dark cycles (Moriyama et al. 2010, Miyagishima et al. 2014). In addition, most of its mitotic kinases are not divergent; for example, the mitotic kinase Aurora kinase in C. merolae is a single-copy gene, whereas almost all land plant species have more than two Aurora kinases (Kawabe et al. 2005, Kato et al. 2011). Taking advantage of these features, we studied a PLK ortholog in C. merolae, CmPLK.

\section{Materials and methods}

\section{Cell strain and culture}

C. merolae strain 10D was used in this study. Cell culture was performed as described previously (Kato et al. 2011).

Sequence alignment and phylogenetic tree construction

PLK orthologs were collected using BLASTP and identified by the kinase domain and PBDs. We used the following PLK orthologs: HsPLK1-5 in Homo sapiens (NCBI reference sequences: NP_005021.2, NP_006613.2, NP_004064.2, NP_055079.3, NP 001230008.1), RnPlk1-5 in Rattus norvegicus (NP 058796.1, NP 114009.1, NP_071523.1， NP_001101139.1， NP_001164028.1), Dmpolo and DmSĀK in Drosophila melanogaster (NP_001014592.1, NP_649324.1), Spplo1 in Schizosaccharomyces pombe (NP593647.1), ScCDC5 in Saccharomyces cerevisiae (NP_013714.1), DdPlk in Dictyostelium discoideum (XP_644113.1), Drplk1, 2a, 2b, 3-4 in Danio rerio (NP_001003890.3, XP_003199325.1, NP_001092715.1, NP 9558465.1, NP_001112364.1), Ceplk-1-3 in Caenorhabditis elegans (NP_001021174.1, NP 491036.1, NP 501196.1), CmPLK in Cyanidioschyzon merolae (XP_005535647.1), OtPLK in Ostreococcus tauri (XP_003078598.1), BfPLK1 and BfPLK4 in Branchiostoma floridae (XP 002591211.1, XP 002585531.1), KfPLK in Klebsormidium flaccidum (GenBank: GAQ79882.1), CrPLK in Chlamydomonas reinhardtii (Protein name in phytozome11: Cre03.g190050.t1.1.p), VcPLK in Volvox carteri (Protein name in phytozome11: Vocar.0035s0030.1.p), and GsPLK in Galdieria sulphuraria (NCBI reference sequence: XP_005704690.1). The amino acid sequences of the PLKs were aligned using
CLC Main Workbench Version 7.7.3. A phylogenetic tree was constructed by the Neighbor-Joining method with CLC Main Workbench Version 7.7.3. using NEK11 in Homo sapiens (NCBI Reference Sequence: NP_079076.3) as an outgroup. Bootstrap probabilities for each node were calculated with 1000 replicates.

\section{Plasmid construction}

The CmPLK-sGFP expression vector (pTH2PLCmPLK-sGFP) was constructed using a DNA fragment including the $5^{\prime}$ flanking region $(2 \mathrm{~kb})$ and the coding region, which was amplified with the primers Cmplk trangent_F (5'-tgcaagcttgcagaaaagccaggaatccg- $\left.3^{\prime}\right)$ and Cmplk_trangent_R (5'-tctctagagacttgcgctggcggaa- $\left.3^{\prime}\right)$. The amplified fragment was subcloned into the multicloning site between the HindIII and XbaI sites of the pTH-2PL vector (Ohnuma et al. 2009). The expression vector for the CmPLK recombinant protein (pET300 NT_DEST_CmPLK) was constructed using the Gateway technology (Thermo Fisher Scientific, Waltham, MA, USA). The coding region of CmPLK was amplified with the primers PLK_entry_F (5'-cac cat ggetttcttggg cct ccg-3') and PLK_entry_R (5'-gacttg cgctggcggaac cg-3'), subcloned into the pENTR ${ }^{\mathrm{TM}} / \mathrm{D}-\mathrm{TOPO}^{\circledR}$ vector (Thermo Fisher Scientific) and cloned into pET300/NT-DEST (Thermo Fisher Scientific).

\section{Transformation}

Using $10 \mu \mathrm{g}$ of pTH2PL-CmPLK-sGFP, C. merolae cells were transformed according to the methods of Ohnuma et al. (2014)

\section{Preparation of the recombinant protein}

pET300_NT_DEST_CmPLK was transformed into BL21 Escherichia coli and expression of the recombinant proteins was induced by $1.0 \mathrm{mM}$ isopropyl-betad-thiogalactopyranoside. After cultivation for $3 \mathrm{~h}$ at $37^{\circ} \mathrm{C}$, the protein-expressing cells were harvested and homogenized by sonication. The insoluble fraction was washed with Wash buffer I ( $20 \mathrm{mM}$ Tris- $\mathrm{HCl} \mathrm{pH} 8.0$, $500 \mathrm{mM} \mathrm{NaCl}, 0.5 \%$ Triton X-100) and eluted with His-binding buffer $(8 \mathrm{M}$ urea, $20 \mathrm{mM}$ phosphate buffer, $500 \mathrm{mM} \mathrm{NaCl}, 20 \mathrm{mM}$ imidazole). The cell extract was purified with HisTrap ${ }^{\mathrm{TM}}$ HP (GE Healthcare UK Ltd., Buckinghamshire, UK) with Wash buffer II (6M urea, $20 \mathrm{mM}$ Tris- $\mathrm{HCl}$ pH $8.0,500 \mathrm{mM} \mathrm{NaCl}, 20 \mathrm{mM}$ imidazole, $1 \mathrm{mM}$ 2-mercaptoethanol), Refolding buffer $(20 \mathrm{mM}$ Tris- $\mathrm{HCl} \mathrm{pH} 8.0,500 \mathrm{mM} \mathrm{NaCl}, 20 \mathrm{mM}$ imidazole, $1 \mathrm{mM}$ 2-mercaptoethanol) with urea and Elution buffer I (20 mM Tris- $\mathrm{HCl} \mathrm{pH} 8.0,500 \mathrm{mM} \mathrm{NaCl}, 500 \mathrm{mM}$ imidazole, $1 \mathrm{mM}$ 2-mercaptoethanol).

\section{Preparation of the polyclonal antibody}

To generate the polyclonal antibody against CmPLK, a recombinant protein of the amino acid residues from 600 to 750 of CmPLK (881 amino acids) with $6 \times$ His-tag 
at N-terminal was expressed in NiCo21 (DE3) E. coli. The recombinant protein was purified with HisTrap ${ }^{\mathrm{TM}}$ HP (GE Healthcare UK Ltd.). The purified protein was inoculated into a rabbit and the antibody was purified by affinity chromatography.

\section{Immunoblotting}

The cell pellet was prepared by centrifuging the cell culture and resuspended with TBS $(20 \mathrm{mM}$ Tris- $\mathrm{HCl} \mathrm{pH}$ $7.5,150 \mathrm{mM} \mathrm{NaCl})$. After centrifugation and removal of the supernatant, the pellet was quenched with liquid nitrogen and stored at $-80^{\circ} \mathrm{C}$. Immediately before immunoblotting, the stored pellet was resuspended with distilled water and used as a cell lysate for immunoblotting. Primary and secondary antibodies were used at the following concentrations: $1: 1000$ for the CmPLK antibody, $1: 10000$ for Anti-IgG (H+L chain) (Rabbit) pAb-HRP (MBL, Nagoya, Japan).

\section{Immunofluorescence microscopy}

Cell fixation and immunofluorescence microscopy were performed as described previously (Nishida et al. 2004). Primary and secondary antibodies were used at the following concentrations: 1:50 for the CmPLK antibody, 1:1000 for monoclonal anti- $\alpha$-tubulin clone B-5-1-2 produced in mouse, ascites fluid (Sigma-Aldrich, St. Louis, MO, USA), 1 : 1000 for Alexa-488 donkey anti-Rabbit IgG antibody (Thermo Fisher Scientific), and $1: 1000$ for Alexa-546 goat anti-Mouse IgG antibody (Thermo Fisher Scientific). After immunostaining, the DNA in the cell preparation was stained with $10 \mu \mathrm{g} \mathrm{mL}^{-1}$ 4',6-diamidino-2-phenylindole (DAPI, Roche Diagnostics GmbH, Penzberg, Germany). Immunostained samples were observed under a fluorescence microscope (BX53, Olympus, Tokyo, Japan) equipped with Mirror Unit Optics: U-FUW (Olympus), U-FBNA (Olympus), U-FGW (Olympus), and XF405 (Omega, Brattleboro, VT, USA). Images were acquired with a digital camera (DP72, Olympus) or monochrome camera (GS3-U350S5M-C, Edmund Optics, Barrington, NJ, USA). The images were analyzed using ImageJ (https://imagej.nih. gov/ij/).

\section{Results and discussion}

Our alignment analyses demonstrated that CmPLK had high similarity in both the N-terminal serine/threonine kinase catalytic domain and PBDs to PLK orthologs in the PLK1 subfamily (Fig. 1). Both Thr210 and Ser137, which are responsible for the activation of the human PLK1 kinase domain (Zitouni et al. 2014), were conserved in CmPLK. Phylogenetic analyses showed that CmPLK belonged to the PLK1 subfamily (Fig. 2). These results suggest CmPLK is a homolog of PLK1 in eukaryotic species. We identified a PLK ortholog in a filamentous terrestrial alga, Klebsormidium flaccidum, as KfPLK through a BLASTP search using the sequences of the N-terminal serine/threonine kinase catalytic domain and PBDs. However, we could not find any PLK orthologs or pseudogenes in two flowering plants, Arabidopsis thaliana and Oryza sativa, or two mosses, Marchantia polymorpha and Physcomitrella patens. Considering that $K$. flaccidum can adapt to both fresh water and land environments, this suggests that plants would have evolutionally lost the PLK ortholog before the divergence of land plants.

Our phylogenetic analyses indicated that two PLK orthologs in O. tauri (OtPLK) and G. sulphuraria (GsPLK) belonged to the PLK1 subfamily, whereas KfPLK and two PLK orthologs in $C$. reinhardtii (CrPLK) and $V$. carteri (VcPLK) belonged to the PLK4/SAK subfamily (Fig. 2). In a previous analysis by Carvalho-Santos et al. (2010), CrPLK was placed in the PLK1 subfamily. This difference is a result of the sequences used for phylogenetic analysis; Carvalho-Santos et al. used only the kinase domain sequences, whereas we used full-length sequences. Taken together, these phylogenetic analyses suggest that the plant PLK ancestor was a PLK1-like protein that evolved into a PLK4-like protein during the divergence of green algae, and that this PLK4-like protein disappeared during the land colonization process.

To determine the function of the plant PLK ancestor, we examined the subcellular localization of CmPLK. Using a polyethylene glycol transformation method (Ohnuma et al. 2014), we analyzed the transient expression of CmPLK-sGFP (Fig. 3A). GFP signals were detected in the spindle poles of mitotic cells. To confirm this subcellular localization, we prepared a polyclonal antibody against CmPLK. Western blotting demonstrated that the antibody specifically recognized the recombinant CmPLK protein tagged with a His-tag (MW $99 \mathrm{kDa}$ ) and the endogenous CmPLK protein (MW $96 \mathrm{kDa}$ ) in cell extracts of C. merolae (Fig. 3B). Next, we performed immunostaining experiments for mitotic cells with the antibody against CMPLK and an antibody against $\alpha$-tubulin. In $C$. merolae, the mitochondrial polar microtubules are localized along the mitochondrion between spindle poles (Imoto et al. 2010). CmPLK signals were detected in the spindle poles and on the mitochondrial polar microtubules (Fig. 3C). These localization analyses suggest that CmPLK functions in the spindle poles and mitochondrial polar microtubules. The single Aurora kinase in $C$. merolae is also localized in the spindle poles (Kato et al. 2011, Yoshida et al. 2013), implying that these mitotic kinases regulate the formation of mitotic spindles in a coordinated manner. After the evolutionary disappearance of the PLK ortholog, a paralog of Aurora kinase would have assumed the function of the PLK ortholog in land plants. 


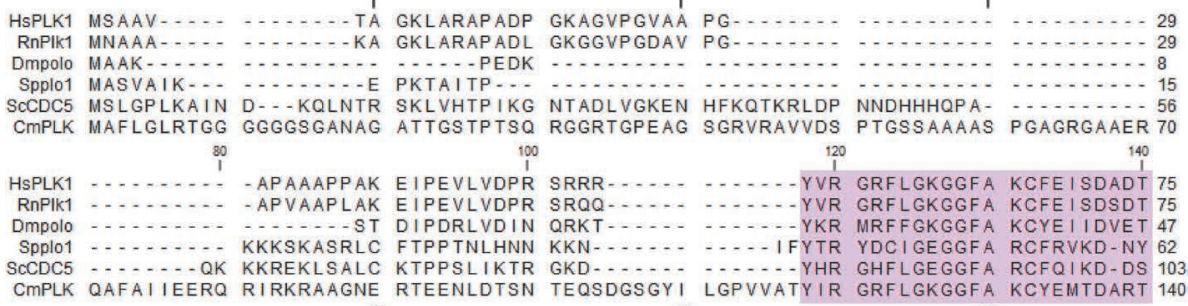
160

180 ${ }_{1}^{200} *$

HSPLK1 KEVFAGKIVP KSLLLKPHQR EKMSMEISIH RSLAHQHVVG FHGFFEDNDF VFVVLELCRR RSLLELHKRR 145 RnPIK1 KEVFPGK IVP KSLLLKPHOK EKMSMETS IH RSLEHQHVVG FHGFFEDSDF VFVVLELCRR RSLLELHKRR 145 DMpolo DDVFAGK IVS KKLMIKHNOK EKTAQEITIH RSLNHPNIVK FHNYFEDSON IYIVLELCKK RSMMELHKRR 117 Spplo1 GN I YAAKV IA KRSLQNDKTK LKLFGE IKVH QSMSHPN IVG FIDCFEDSTN IYL I LELCEH KS LMELLRKR 132 SCCDC5 GEIFAAKTVA KASIKSEKTR KKLLSEIQIH KSMSHPNIVQ FIDCFEDDSN VYILLEICPN GSLMELLKRR 173 CMPLK QRVYAGKIID KSTVTKLRAR QKLRSEIQIH GSLRHPHIVR FEHFFEDEDH VYILLELCDA QSMMELLKRR 210 220 240
1 280 * $\quad 280$

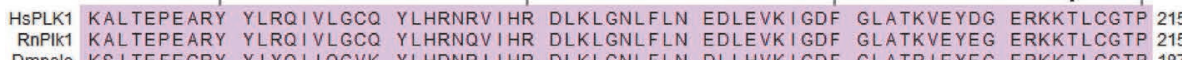
YIYQIIQGVK YLHDNRIIHR DLKLGNLFLN DLLHVKIGDF GLATRIEYEG ERKKTLCGTP 18 Spplo1 KOLTEPEVRY LMMQILGALK YMHKKRVIHR DLKLGNIMLD ESNNVK IGDF GLAALLMDDE ERKMT I CGTP 202 SCCDC5 KVLTEPEVRF FTTQICGAIK YMHSRRVIHR DLKLGNIFFD SNYNLKIGDF GLAAVLANES ERKYT ICGTP 243 CMPLK KRLTEPECRY FMMQILEAVE YMHRNRV IHR DIKLGNLFLT SDLQIKIGDF GLAAKLEYDN ERKRTMCGTP 280 300 320 340
1

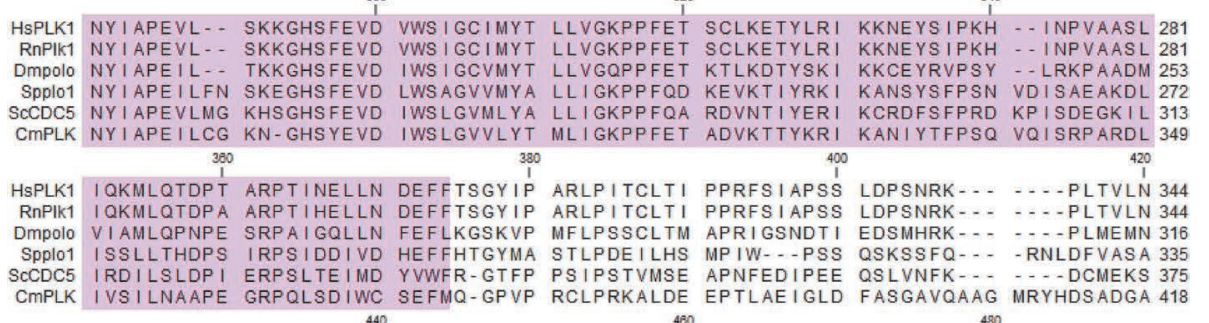

$\stackrel{400}{1}$ 480

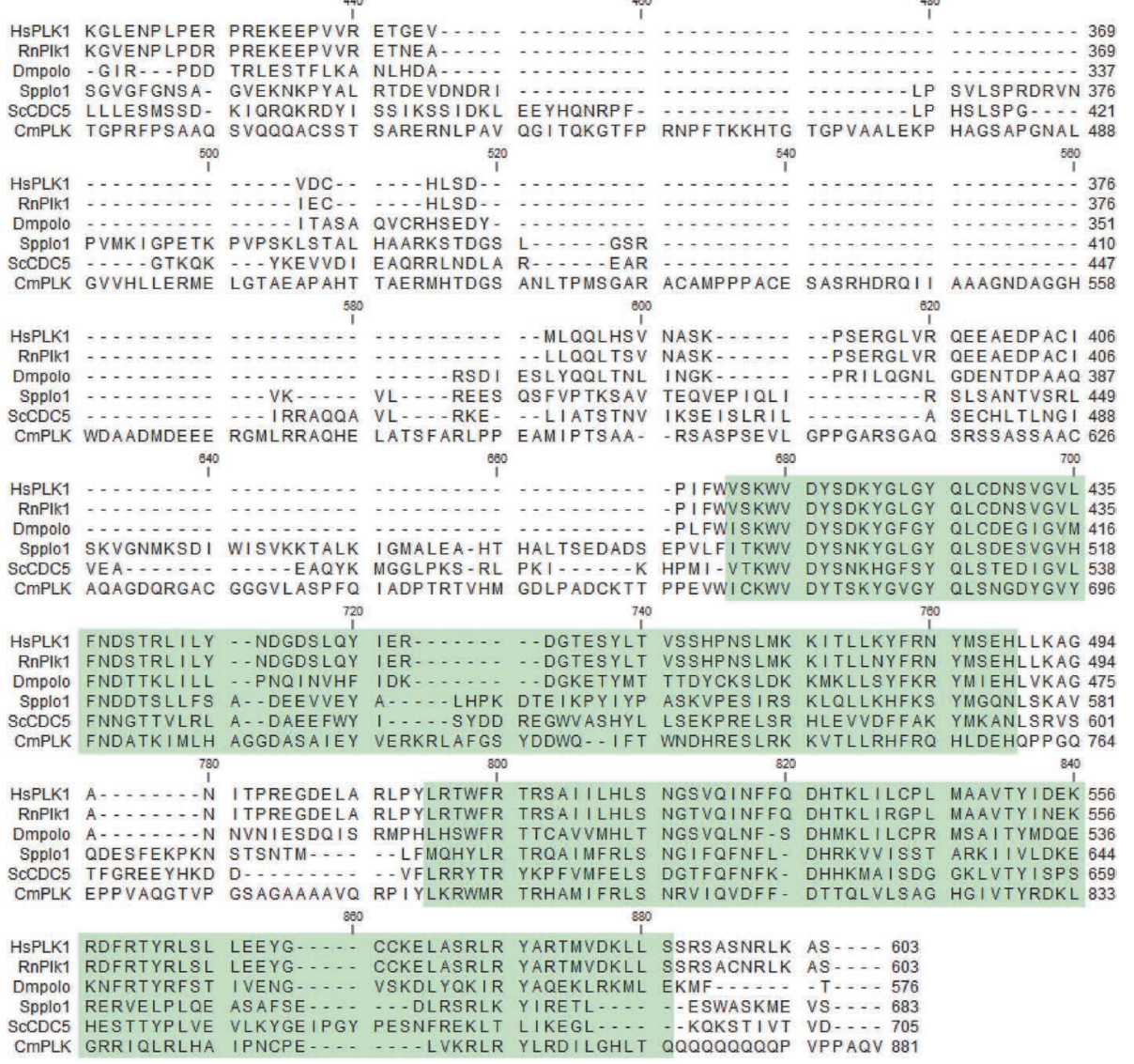

Fig. 1. Alignment of PLK orthologs in the PLK1 subfamily.

The full-length amino acid sequences of HsPLK1 in Homo sapiens, RnPlk1 in Rattus norvegicus, Dmpolo in Drosophila melanogaster, Spplo1 in Schizosaccharomyces pombe, ScCDC5 in Saccharomyces cerevisiae, and CmPLK in Cyanidioschyzon merolae were used for the alignment. Pink and green boxes show the kinase domain and PBDs, respectively. Asterisks indicate amino acids responsible for kinase activation. 


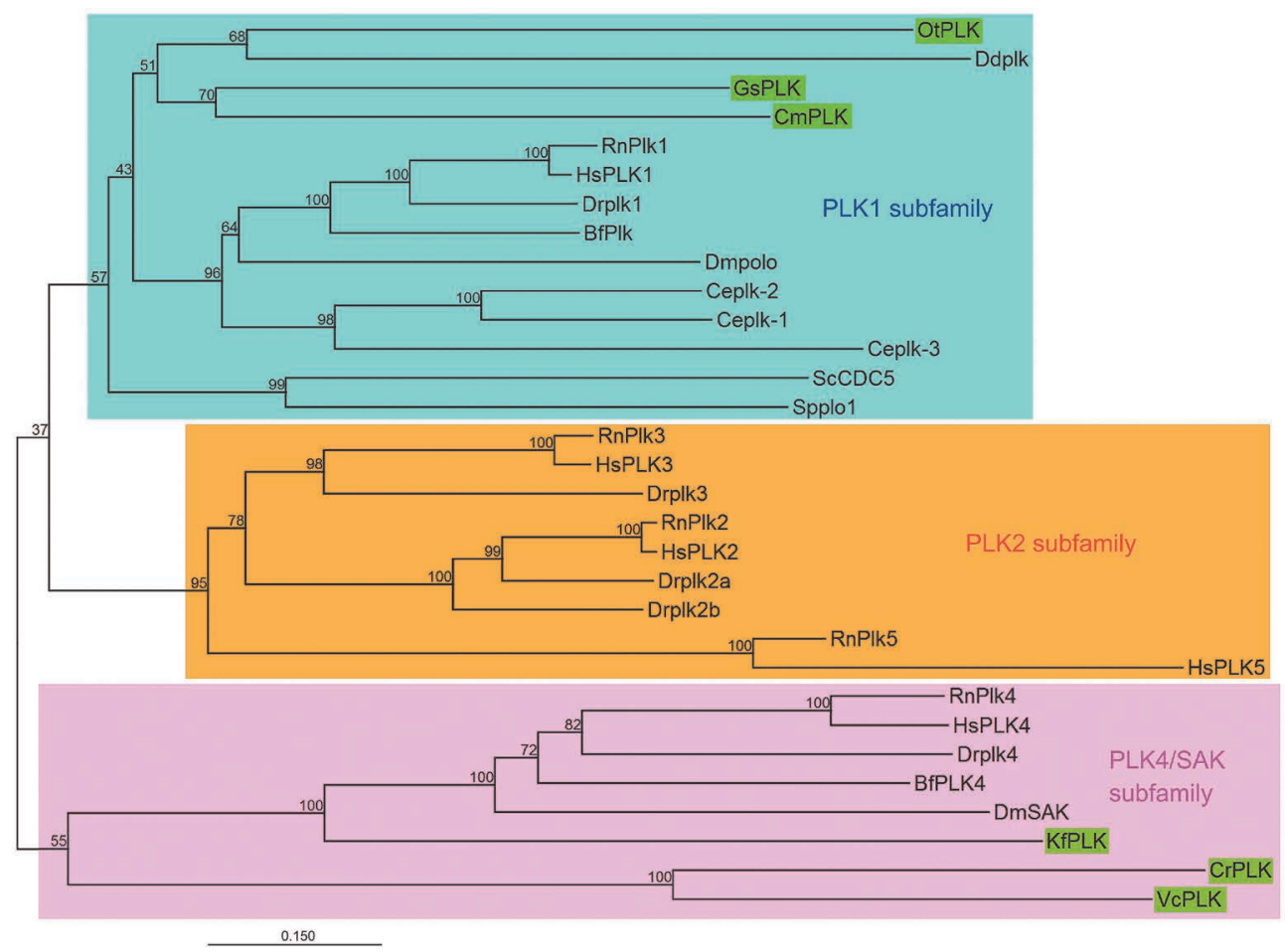

Fig. 2. Phylogenetic tree of PLK orthologs.

A rooted tree was constructed by the Neighbor-Joining method with 1000 bootstrap replicates. The numbers indicate bootstrap values. The sequences include HsPLK1-5 in Homo sapiens, RnPlk1-5 in Rattus norvegicus, Dmpolo and DmSAK in Drosophila melanogaster, Spplo1 in Schizosaccharomyces pombe, ScCDC5 in Saccharomyces cerevisiae, Ddplk in Dictyostelium discoideum, Drplk1, Drplk2a, Drplk2b, Drplk3 and Drplk4 in Danio rerio, Ceplk-1, Ceplk-2, and Ceplk-3 in Caenorhabditis elegans, CmPLK in Cyanidioschyzon merolae, OtPLK in Ostreococcus tauri, BfPLK1 and BfPLK4 in Branchiostoma floridae, KfPLK in Klebsormidium flaccidum, CrPLK in Chlamydomonas reinhardtii, VcPLK in Volvox carteri, and GsPLK in Galdieria sulphuraria. Light blue, orange and pink boxes indicate the PLK1, PLK2 and PLK4/SAK subfamilies, respectively. Green boxes indicate algae.

A

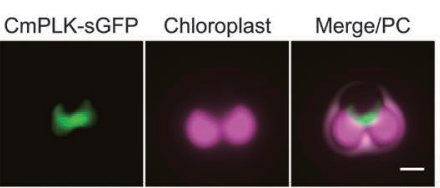

B

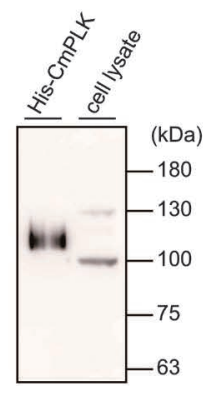

C

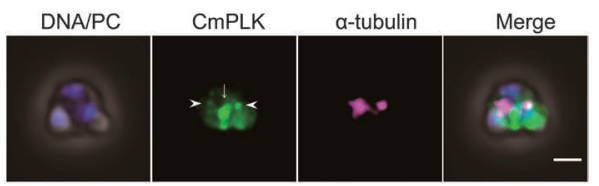

Fig. 3. Subcellular localization of the PLK ortholog in C. merolae.

(A) Expression of CmPLK-sGFP in a mitotic cell of C. merolae. Fluorescence signals were observed without cell fixation after the transformation. The left, middle and right panels show GFP signals, autofluorescence of chloroplasts and merged phase contrast images, respectively. The scale bar indicates $1 \mu \mathrm{m}$. (B) Immunoblot of His-tagged CmPLK recombinant protein and cell lysate with a polyclonal antibody against CmPLK. (C) Immunofluorescence of CmPLK in a mitotic cell of $C$. merolae. The first, second, third and fourth panels show DAPI fluorescence with phase contrast, immunofluorescence of CmPLK, immunofluorescence of $\alpha$-tubulin and merged phase contrast images, respectively. White arrowheads and the arrow indicate spindle poles and mitochondrial polar microtubules, respectively. The scale bar indicates $2 \mu \mathrm{m}$.

\section{Acknowledgements}

This research was supported by a Grant-in-Aid for the X-ray Free Electron Laser Priority Strategy Program (MEXT) to S.M. and MEXT/JSPS KAKENHI to S.M. (15K21750, 15H05962, 15H05955, 15H01238, 26291067).

\section{References}

Andrysik, Z., Bernstein, W. Z., Deng, L., Myer, D. L., Li, Y. Q., Tischfield, J. A., Stambrook, P. J. and Bahassi, E. M. 2010. The novel mouse Polo-like kinase 5 responds to DNA damage and localizes in the nucleolus. Nucleic Acids Res. 38: 2931-2943.

Barr, F. A., Silljé, H. H. W. and Nigg, E. A. 2004. Polo-like kinases and the orchestration of cell division. Nat. Rev. Mol. Cell Biol. 
5: 429-441.

Bettencourt-Dias, M., Rodrigues-Martins, A., Carpenter, L., Riparbelli, M., Lehmann, L., Gatt, M. K., Carmo, N., Balloux, F., Callaini, G. and Glover, D. M. 2005. SAK/PLK4 is required for centriole duplication and flagella development. Curr. Biol. 15: 2199-2207.

de Cárcer, G., Escobar, B., Higuero, A. M., García, L., Ansón, A., Pérez, G., Mollejo, M., Manning, G., Meléndez, B., Abad-Rodríguez, J. and Malumbres, M. 2011a. Plk5, a polo box domain-only protein with specific roles in neuron differentiation and glioblastoma suppression. Mol. Cell. Biol. 31: 1225-1239.

de Cárcer, G., Manning, G. and Malumbres, M. 2011b. From Plk1 to Plk5: Functional evolution of polo-like kinases. Cell Cycle 10: 2255-2262.

Carvalho-Santos, Z., Machado, P., Branco, P., Tavares-Cadete, F., Rodrigues-Martins, A., Pereira-Leal, J. B. and Bettencourt-Dias, M. 2010. Stepwise evolution of the centriole-assembly pathway. J. Cell Sci. 123: 1414-1426.

Cizmecioglu, O., Warnke, S., Arnold, M., Duensing, S. and Hoffmann, I. 2008. Plk2 regulated centriole duplication is dependent on its localization to the centrioles and a functional polo-box domain. Cell Cycle 7: 3548-3555.

Dewitte, W. and Murray, J. A. H. 2003. The plant cell cycle. Annu. Rev. Plant Biol. 54: 235-264.

Fode, C., Motro, B., Yousefi, S., Heffernan, M. and Dennis, J. W. 1994. Sak, a murine protein-serine/threonine kinase that is related to the Drosophila polo kinase and involved in cell proliferation. Proc. Natl. Acad. Sci. U.S.A. 91: 6388-6392.

Hodges, M. E., Scheumann, N., Wickstead, B., Langdale, J. A. and Gull, K. 2010. Reconstructing the evolutionary history of the centriole from protein components. J. Cell Sci. 123: 1407-1413.

Imoto, Y., Fujiwara, T., Yoshida, Y., Kuroiwa, H., Maruyama, S. and Kuroiwa, T. 2010. Division of cell nuclei, mitochondria, plastids, and microbodies mediated by mitotic spindle poles in the primitive red alga Cyanidioschyzon merolae. Protoplasma 241: 63-74.

Imoto, Y., Kuroiwa, H., Yoshida, Y., Ohnuma, M., Fujiwara, T., Yoshida, M., Nishida, K., Yagisawa, F., Hirooka, S., Miyagishima, S., Misumi, O., Kawano, S. and Kuroiwa, T. 2013. Singlemembrane-bounded peroxisome division revealed by isolation of dynamin-based machinery. Proc. Natl. Acad. Sci. U.S.A. 110: 9583-9588.

Kato, S., Imoto, Y., Ohnuma, M., Matsunaga, T. M., Kuroiwa, H., Kawano, S., Kuroiwa, T. and Matsunaga, S. 2011. Aurora kinase of the red alga Cyanidioschyzon merolae is related to both mitochondrial division and mitotic spindle formation. Cytologia 76: 455-462.

Kawabe, A., Matsunaga, S., Nakagawa, K., Kurihara, D., Yoneda, A., Hasezawa, S., Uchiyama, S. and Fukui, K. 2005. Characterization of plant Aurora kinases during mitosis. Plant Mol. Biol. 58: $1-13$.

Kleylein-Sohn, J., Westendorf, J., Le Clech, M., Habedanck, R., Stierhof, Y. D. and Nigg, E. A. 2007. Plk4-Induced centriole biogenesis in human cells. Dev. Cell 13: 190-202.

Lowery, D. M., Lim, D. and Yaffe, M. B. 2005. Structure and function of polo-like kinases. Oncogene 24: 248-259.

Ma, S., Charron, J. and Erikson, R. L. 2003. Role of Plk2 (Snk) in mouse development and cell proliferation. Mol. Cell. Biol. 23: 6936-6943.

Matsuzaki, M., Misumi, O., Shin-I, T., Maruyama, S., Takahara, M., Miyagishima, S., Mori, T., Nishida, K., Yagisawa, F., Nishida, K., Yoshida, Y., Nishimura, Y., Nakao, S., Kobayashi, T., Momoyama, Y., Higashiyama, T., Minoda, A., Sano, M., Nomoto, H., Oishi, K., Hayashi, H., Ohta, F., Nishizaka, S., Haga, S.,
Miura, S., Morishita, T., Kabeya, Y., Terasawa, K., Suzuki, Y., Ishii, Y., Asakawa, S., Takano, H., Ohta, N., Kuroiwa, H., Tanaka, K., Shimizu, N., Sugano, S., Sato, N., Nozaki, H., Ogasawara, N., Kohara, Y. and Kuroiwa, T. 2004. Genome sequence of the ultrasmall unicellular red alga Cyanidioschyzon merolae 10D. Nature 428: 653-657.

Miyagishima, S., Fujiwara, T., Sumiya, N., Hirooka, S., Nakano, A., Kabeya, Y. and Nakamura, M. 2014. Translation-independent circadian control of the cell cycle in a unicellular photosynthetic eukaryote. Nat. Commun. 5: 3807.

Moriyama, T., Terasawa, K., Sekine, K., Toyoshima, M., Koike, M., Fujiwara, M. and Sato, N. 2010. Characterization of cell-cycledriven and light-driven gene expression in a synchronous culture system in the unicellular rhodophyte Cyanidioschyzon merolae. Microbiology 156: 1730-1737.

Nishida, K., Misumi, O., Yagisawa, F., Kuroiwa, H., Nagata, T. and Kuroiwa, T. 2004. Triple immunofluorescent labeling of FtsZ, dynamin, and EF-Tu reveals a loose association between the inner and outer membrane mitochondrial division machinery in the red alga Cyanidioschyzon merolae. J. Histochem. Cytochem. 52: 843-849.

Nozaki, H., Takano, H., Misumi, O., Terasawa, K., Matsuzaki, M., Maruyama, S., Nishida, K., Yagisawa, F., Yoshida, Y., Fujiwara, T., Takio, S., Tamura, K., Chung, S. J., Nakamura, S., Kuroiwa, H., Tanaka, K., Sato, N. and Kuroiwa, T. 2007. A 100\%-complete sequence reveals unusually simple genomic features in the hotspring red alga Cyanidioschyzon merolae. BMC Biol. 5: 28.

Nurse, P. 1990. Universal control mechanism regulating onset of Mphase. Nature 344: 503-508.

Ohnuma, M., Misumi, O., Fujiwara, T., Watanabe, S., Tanaka, K. and Kuroiwa, T. 2009. Transient gene suppression in a red alga, $C y$ anidioschyzon merolae 10D. Protoplasma 236: 107-112.

Ohnuma, M., Yokoyama, T., Inouye, T., Sekine, Y., Kuroiwa, T. and Tanaka, K. 2014. Optimization of polyethylene glycol (PEG)mediated DNA introduction conditions for transient gene expression in the unicellular red alga Cyanidioschyzon merolae. J. Gen. Appl. Microbiol. 60: 156-159.

Ohta, N., Matsuzaki, M., Misumi, O., Miyagishima, S. Y., Nozaki, H., Tanaka, K., Shin-I, T., Kohara, Y. and Kuroiwa, T. 2003. Complete sequence and analysis of the plastid genome of the unicellular red alga Cyanidioschyzon merolae. DNA Res. 10: 67-77.

Ohta, N., Sato, N. and Kuroiwa, T. 1998. Structure and organization of the mitochondrial genome of the unicellular red alga $C y$ anidioschyzon merolae deduced from the complete nucleotide sequence. Nucleic Acids Res. 26: 5190-5198.

Sunkel, C. E. and Glover, D. M. 1988. polo, a mitotic mutant of Drosophila displaying abnormal spindle poles. J. Cell Sci. 89: 25-38.

Wang, Q., Xie, S., Chen, J., Fukasawa, K., Naik, U., Traganos, F., Darzynkiewicz, Z., Jhanwar-Uniyal, M. and Dai, W. 2002. Cell cycle arrest and apoptosis induced by human polo-like kinase 3 is mediated through perturbation of microtubule integrity. Mol. Cell. Biol. 22: 3450-3459

Yoshida, Y., Fujiwara, T., Imoto, Y., Yoshida, M., Ohnuma, M., Hirooka, S., Misumi, O., Kuroiwa, H., Kato, S., Matsunaga, S. and Kuroiwa, T. 2013. The kinesin-like protein TOP promotes Aurora localisation and induces mitochondrial, chloroplast and nuclear division. J. Cell Sci. 126: 2392-2400.

Zimmerman, W. C. and Erikson, R. L. 2007. Polo-like kinase 3 is required for entry into S phase. Proc. Natl. Acad. Sci. U.S.A. 104: $1847-1852$

Zitouni, S., Nabais, C., Jana, S. C., Guerrero, A. and BettencourtDias, M. 2014. Polo-like kinases: Structural variations lead to multiple functions. Nat. Rev. Mol. Cell Biol. 15: 433-452. 